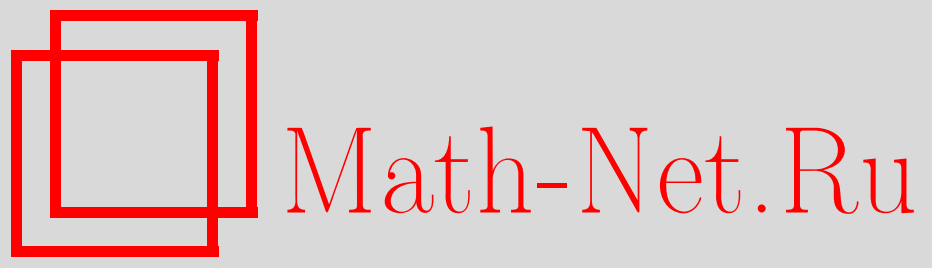

А. А. Заикин, Дефект размера нерандомизированного критерия и влияние рандомизации на сокращение необходимого объема выборки при тестировании вероятности успеха в схеме испытаний Бернулли, Теория вероятн. и ее примен., 2014, том 59, выпуск 3, 417-435

DOI: https://doi.org/10.4213/tvp4577

Использование Общероссийского математического портала Math-Net.Ru подразумевает, что вы прочитали и согласны с пользовательским соглашением http://www . mathnet.ru/rus/agreement

Параметры загрузки:

IP : 54.224 .187 .69

26 апреля 2023 г., 15:39:05

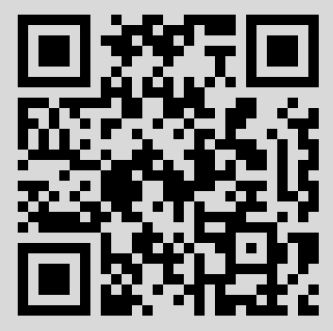


ТЕОРИЯ ВЕРОЯТНОСТЕЙ

И ЕЕ ПРИМЕНЕНИЯ

Основан в январе 1956 г.

Том 59

Выходит 4 раза в год

Москва • Наука

Выпуск 3, 2014 год июль, август, сентябрь

(C) 2014 г.

ЗАИКИН А. А.*

\title{
ДЕФЕКТ РАЗМЕРА НЕРАНДОМИЗИРОВАННОГО КРИТЕРИЯ И ВЛИЯНИЕ РАНДОМИЗАЦИИ НА СОКРАЩЕНИЕ НЕОБХОДИМОГО ОБЪЕМА ВЫБОРКИ ПРИ ТЕСТИРОВАНИИ ВЕРОЯТНОСТИ УСПЕХА В СХЕМЕ ИСПЫТАНИЙ БЕРНУЛЛИ
}

\begin{abstract}
В задаче проверки гипотезы о значении вероятности успеха в испытаниях Бернулли устанавливается верхняя асимптотическая (объем выборки $n \rightarrow \infty$ ) граница для разности между размером нерандомизированного наиболее мощного критерия и предписанным уровнем значимости. Находится асимптотика редукции числа наблюдений, необходимых для различения двух близких гипотез о вероятности успеха с заданными ограничениями на вероятности ошибок первого и второго рода, когда вместо нерандомизированного критерия используется рандомизированный наиболее мощный критерий. Проводится сравнительный анализ трех известных асимптотических формул для необходимого объема выборки и предлагаются их модификации, обладающие большей точностью.

Ключевые слова и фразы: биномиальное распределение, близкие гипотезы, асимптотики необходимого объема выборки, разложение Корниша-Фишера, дефект размера нерандомизированного критерия, эффект рандомизации.
\end{abstract}

Введение. Рассматривается схема испытаний Бернулли с вероятностью успеха $p$, относительно значения которой высказываются две конкурирующие гипотезы: $H_{0}: p \leqslant p_{0}$ и $H_{1}: p \geqslant p_{1}\left(>p_{0}\right)$. Для различения этих гипотез по наблюдениям случайной выборки $X_{1}, \ldots, X_{n}$ фиксированного объема $n$ обычно используется равномерно наиболее мощный нерандомизированный критерий, основанный на статистике $T=\sum_{k=1}^{n} X_{k}$, которая имеет биномиальное распределение с параметрами $n$ и $p$. При заданном уровне значимости $\alpha$ критерий отвергает нулевую гипотезу $H_{0}$, если наблюдаемое значение статистики $T$ превосходит значение критической константы $C_{n}(\alpha)$, которая определяется как

*Казанский федеральный университет, Казань, Россия; e-mail: kaskun@gmail.com 
наименьшее значение $C$, удовлетворяющее неравенству $\mathbf{P}_{0}\{T \geqslant C\} \leqslant \alpha$ (здесь и далее $\mathbf{P}_{i}$ означает, что вероятность вычисляется по биномиальному распределению с параметром $\left.p=p_{i}, i=0,1\right)$. В силу дискретности распределения статистики $T$ зачастую не представляется возможным подобрать $C_{n}(\alpha)$ таким образом, чтобы размер критерия был в точности равен заданному уровню $\alpha$. Вывод асимптотической $(n \rightarrow \infty)$ верхней границы для разности (деØекта размера нерандомизированного критерия) $D(n)=D\left(n ; \alpha, p_{0}\right)=\alpha-\mathbf{P}_{0}\left(T \geqslant C_{n}(\alpha)\right)$ представляет одну из задач, решаемых в данной статье.

Другая, решаемая в статье задача, связана с оптимальным планированием объема испытаний при различении гипотез $H_{0}$ и $H_{1}$. Если используется нерандомизированный критерий, то при заданных ограничениях $\alpha$ и $\beta$ на вероятности ошибок первого и второго рода минимальный объем наблюдений определяется посредством задания целочисленной критической константы $C_{n}$ и объема выборки $n=n(\alpha, \beta)$, удовлетворяющих неравенствам

$$
\mathbf{P}_{0}\left\{T \geqslant C_{n}\right\} \leqslant \alpha, \quad \mathbf{P}_{1}\left\{T<C_{n}\right\} \leqslant \beta .
$$

Определенный таким способом минимальный объем наблюдений, гарантирующий заданные ограничения $(\alpha, \beta)$ на вероятности ошибочных решений при использовании нерандомизированного критерия $T \geqslant C$, в дальнейшем будет называться требуемым объемом наблюдений (сокращенно - ТОН).

Называть ТОН необходимым объемом выборки некорректно. Дело в том, что в математической статистике необходимым объемом выборки (сокращенно - НОВ) называется минимальный объем наблюдений $n^{*}=n^{*}(\alpha, \beta)$, при котором существует критерий заданной силы $(\alpha, \beta)$. В данном случае такой критерий является рандомизированным и он основан на статистике $T+U$, где $U$ - случайная величина с равномерным на интервале $(0,1)$ распределением. Распределение статистики $T+U$ принадлежит непрерывному типу и НОВ находится как наименьшее целое $n$, удовлетворяющее соотношениям

$$
\mathbf{P}_{0}\left\{T+U \geqslant C_{n}\right\}=\alpha, \quad \mathbf{P}_{1}\left\{T+U<C_{n}\right\} \leqslant \beta .
$$

Использование рандомизированного критерия приводит к сокращению объема наблюдений, гарантирующего заданные ограничения $\alpha$ и $\beta$ на вероятности ошибочных решений. Асимптотическая оценка разности $n(\alpha, \beta)-n^{*}(\alpha, \beta)$ в терминах сближающихся гипотез $\left(\Delta=p_{1}-p_{0} \rightarrow 0\right)-$ вторая из важнейших задач, решаемых в данной статье.

Естественно, было необходимо проанализировать и сравнить известные асимптотические методы оценки ТОН $n(\alpha, \beta)$. Анализ формул показал, что их можно значительно улучшить; предлагаются несколько 
новых методов асимптотической оценки ТОН, обладающих большей точностью.

Оставшаяся часть статьи организована следующим образом. В первом параграфе приводятся асимптотические $(\alpha+\beta \rightarrow 0)$ формулы Володина-Новикова [1] и Чернова [2] для ТОН и предлагается более точный метод асимптотической оценки ТОН с помощью теоремы о больших уклонениях. Приводятся табличные данные по сравнению точностных свойств полученных оценок $n(\alpha, \beta)$ с помощью всех трех методов. Результаты этого параграфа можно рассматривать как ответ на замечание Ширяева (см. [3, с. 142]) о необходимости вывода асимптотик $n(\alpha, \beta)$ с помощью теоремы о больших уклонениях, а не с помощью центральной предельной теоремы.

Во втором параграфе решается проблема оценки ТОН при тестировании очень малых значений вероятности успеха $p$. Приводится алгоритм оценки ТОН, предложенный в статье [4], и показывается, что этот алгоритм можно упростить и свести вывод асимптотической оценки $n(\alpha, \beta)$ к решению достаточно простой системы из двух уравнения.

В третьем параграфе устанавливается асимптотическая $(n \rightarrow \infty)$ верхняя граница для разности между предписанным уровнем значимости нерандомизированного критерия и его размером - находится асимптотика дефекта $D(n)$ размера нерандомизированного критерия. Полученный результат применяется в следующем параграфе для асимптотической оценки $n(\alpha, \beta)$ в схеме сближающихся гипотез на основе разложения Корниша-Фишера. Вывод асимптотического разложения представляет собой распространение метода оценки ТОН, предложенного в статье [5] для тестовых статистик с непрерывным распределением, на случай дискретного распределения тестовой статистики.

Наконец, пятый параграф посвящен выводу асимптотического разложения для $\mathrm{HOB} n^{*}(\alpha, \beta)$ в случае рандомизированного критерия. В силу непрерывности распределения тестовой статистики $T+U$ представляется возможным прямое применение метода статьи [5] с помощью соответствующей модификации разложений Эджворта и КорнишаФишера применительно к распределению статистики $T+U$. Находится асимптотика $\left(\Delta=p_{1}-p_{0} \rightarrow 0\right)$ величины редукции требуемого объема испытаний при использовании рандомизированного критерия в сравнении с его нерандомизированным аналогом. Точность всех полученных формул иллюстрируется численно в широком диапазоне гипотетических значений параметров и ограничений на вероятности ошибок.

1. Аппроксимация ТОН с использованием теоремы о больших уклонениях. Метод асимптотической оценки ТОН $n(\alpha, \beta)$ при малых значениях $\alpha$ и $\beta$ основан на теореме о вероятности больших уклонениях (см. монографию [6, §8 гл. 8], а также статью [7]). 
Рассмотрим, нерандомизированный критерий различения двух простых гипотез, основанный на статистике отношения правдоподобия

$$
L_{n}=\sum_{i=1}^{n} \ln \frac{f_{1}\left(X_{i}\right)}{f_{0}\left(X_{i}\right)}
$$

где $f_{i}, i=0,1,-$ функции плотности $X_{1}$ при справедливости соответствующих гипотез.

Для любой постоянной $C$ в силу теоремы о вероятности больших уклонениях существуют две асимптотические $(n \rightarrow \infty)$ формулы, которые могут быть использованы для асимптотической оценки ТОН критерия $L_{n} \geqslant C$ и соответствующей ТОН $n=n(\alpha, \beta)$ критической константы $C_{n}$ :

$$
\begin{aligned}
\mathbf{P}_{0}\left\{L_{n} \geqslant \ln C\right\} & \sim \frac{1}{\sigma \lambda_{0} \sqrt{2 \pi n}} \exp \left\{-\lambda_{0} \ln C+n \ln \psi\left(\lambda_{0}\right)\right\}, \\
\mathbf{P}_{1}\left\{L_{n}<\ln C\right\} & \sim \frac{1}{\sigma \lambda_{1} \sqrt{2 \pi n}} \exp \left\{\lambda_{1} \ln C+n \ln \psi\left(\lambda_{0}\right)\right\},
\end{aligned}
$$

где

$$
\psi(\lambda)=\mathbf{E}_{0} \exp \left[\lambda \ln \frac{f_{1}(\xi)}{f_{0}(\xi)}\right],
$$

$\lambda_{0}$ - решение уравнения $\psi^{\prime}(\lambda)=0$ и $\sigma^{2}=\psi^{\prime \prime}\left(\lambda_{0}\right) / \psi\left(\lambda_{0}\right)$. На основе этих асимптотических представлений в [1] была получена асимптотическая $(\alpha+\beta \rightarrow 0)$ формула для ТОН:

$$
n(\alpha, \beta) \sim \frac{\min \{\ln \alpha, \ln \beta\}}{\ln \psi\left(\lambda_{0}\right)} .
$$

В случае выбора в схеме Бернулли $f_{i}(\xi)=p_{i}^{\xi}\left(1-p_{i}\right)^{1-\xi}$, где $\xi=1 \mathrm{c}$ вероятностью $p_{i}$ и $\xi=0$ с вероятностью $1-p_{i}, i=0,1$, так что

$$
\psi(\lambda)=\mathbf{E}_{0} \exp \left\{\lambda \ln \frac{f_{1}(\xi)}{f_{0}(\xi)}\right\}=\left(1-p_{1}\right)^{\lambda}\left(1-p_{0}\right)^{1-\lambda}+p_{1}^{\lambda} p_{0}^{1-\lambda},
$$

а производная функции $\psi$

$$
\psi^{\prime}(\lambda)=\left(1-p_{1}\right)^{\lambda}\left(1-p_{0}\right)^{1-\lambda} \ln \frac{1-p_{1}}{1-p_{0}}+p_{1}^{\lambda} p_{0}^{1-\lambda} \ln \frac{p_{1}}{p_{0}} .
$$

Уравнение $\psi^{\prime}(\lambda)=0$ имеет единственное решение

$$
\lambda_{0}=\left[\ln \left(-\ln \frac{1-p_{1}}{1-p_{0}}\right)-\ln \left(\frac{p_{0}}{1-p_{0}} \ln \frac{p_{1}}{p_{0}}\right)\right]\left[\ln \frac{p_{1}\left(1-p_{0}\right)}{p_{0}\left(1-p_{1}\right)}\right]^{-1} .
$$

Нетрудно также видеть, что $\lambda_{1}=1-\lambda_{0}$. 
Преимуществом этой формулы является, очевидно, простота. Однако формула сходится к $n(\alpha, \beta)$, когда $\alpha+\beta \rightarrow 0$ очень медленно, даже при $\alpha=0.01, \beta=0.01$ она не дает удовлетворительных результатов.

Приведем еще одну аппроксимацию для ТОН, полученную Черновым в работе [2] при стремление к нулю лишь одного из ограничений $\alpha=\alpha_{0}$ или $\beta=\alpha_{1}$ :

$$
n(\alpha, \beta) \sim-\frac{\ln \alpha_{1-i}}{\mathbf{I}(i, 1-i)} \quad \text { при } \alpha_{1-i} \rightarrow 0, \alpha_{i}=\text { const },
$$

где

$$
\mathbf{I}(i, 1-i)=\mathbf{E}_{i} \ln \frac{f_{i}(\xi)}{f_{1-i}(\xi)}
$$

- информация по Кульбаку-Лейблеру. В случае различения гипотез о вероятности успеха $p$ по данным наблюдений в схеме Бернулли эта информация равна

$$
\left(1-p_{i}\right) \ln \frac{1-p_{i}}{1-p_{1-i}}+p_{i} \ln \frac{p_{i}}{p_{1-i}}, \quad i=0,1 .
$$

Отметим, что при применении этой формулы более точный результат получается при использовании наименьшего из двух ограничений $\alpha_{0}, \alpha_{1}$ на вероятности ошибок.

Можно несколько уточнить данные асимптотики ТОН, если перейти к системе уравнений, полученных приравниванием правых частей соотношений (3) и (4) соответствующим ограничениям $\alpha$ и $\beta$ на вероятности ошибок:

$$
\begin{aligned}
& \ln \left(\alpha \sigma \lambda_{0} \sqrt{2 \pi n}\right)=-\lambda_{0} \ln C+n \ln \psi\left(\lambda_{0}\right), \\
& \ln \left(\beta \sigma \lambda_{1} \sqrt{2 \pi n}\right)=\lambda_{1} \ln C+n \ln \psi\left(\lambda_{0}\right) .
\end{aligned}
$$

Исключая из этих уравнений $\ln C$, приходим к уравнению для $n$. Чтобы найти асимптотику корня этого уравнения, заменим $\ln n$ его аппроксимацией

$$
\ln n \approx \ln \widetilde{n}+\frac{1}{\widetilde{n}}(n-\widetilde{n})
$$

по формуле Тейлора в окрестности точки

$$
\widetilde{n}=\frac{\min \{\ln \alpha, \ln \beta\}}{\ln \psi\left(\lambda_{0}\right)}
$$

- асимптотики $n(\alpha, \beta)$ по формуле (5). В результате получим линейное уравнение относительно $n$, решение которого устанавливает следующую асимптотику:

$$
\begin{aligned}
n(\alpha, \beta) \sim & {\left[\ln \left(\beta \sigma \lambda_{1} \sqrt{2 \pi}\right)+\frac{\lambda_{1}}{\lambda_{0}} \ln \left(\alpha \sigma \lambda_{0} \sqrt{2 \pi}\right)+\frac{1}{2 \lambda_{0}} \ln \widetilde{n}-\frac{1}{2 \lambda_{0}}\right] } \\
& \times\left[\frac{1}{\lambda_{0}} \ln \psi\left(\lambda_{0}\right)-\frac{1}{2 \lambda_{0} \widetilde{n}}\right]^{-1} .
\end{aligned}
$$


Эта формула асимптотической оценки $n(\alpha, \beta)$ при $\alpha+\beta \rightarrow 0$ естественно применима и для случая выбора в схеме Бернулли с подстановкой выражений для $\psi, \lambda_{0}, \lambda_{1}$ и $\sigma$, полученных после записи формулы (5).

В табл. 1 показаны полученные результаты. В левом верхнем углу каждой ячейки - точное значение ТОН $n(\alpha, \beta)$ нерандомизированного критерия; в правом верхнем - аппроксимация Чернова (6); в левом нижнем - грубое приближение (5); в правом нижнем - полученная здесь формула (7).

Приведенные в таблице данные убедительно демонстрируют хорошую точность в аппроксимации ТОН с помощью новой формулы (7) и показывают, что известные формулы (6) и (5) вряд ли можно использовать даже при очень малых значениях $\alpha$ и $\beta$.

Таблица 1. Значения ТОН с использованием теоремы о больших уклонениях

\begin{tabular}{|c|c|c|c|c|c|}
\hline \multicolumn{6}{|c|}{$\alpha=0.01 ; \beta=0.01$} \\
\hline$p_{0}$ & & 0.2 & 0.3 & 0.4 & 0.5 \\
\hline \multirow{2}{*}{0.1} & & $272 \quad 126$ & 83 & 42 & 25 \\
\hline & & $692 \quad 275$ & $174 \quad 83$ & 41 & 43 \\
\hline \multirow{2}{*}{0.2} & $479 \quad 189$ & & $402 \quad 179$ & 111 & 52 \\
\hline & $943 \quad 477$ & & $1096 \quad 412$ & $250 \quad 113$ & 104 \\
\hline \multirow{2}{*}{0.3} & $\begin{array}{ll}143 & 60\end{array}$ & $\begin{array}{ll}713 & 269\end{array}$ & & $495 \quad 214$ & 130 \\
\hline & $249 \quad 143$ & $1473 \quad 716$ & & 1371502 & 296 \\
\hline \multirow{2}{*}{0.4} & $\begin{array}{ll}73 & 31\end{array}$ & $\begin{array}{ll}196 & 76\end{array}$ & $867 \quad 320$ & & 535 \\
\hline & 115 & $353 \quad 196$ & $1831 \quad 873$ & & $1510 \quad 547$ \\
\hline \multirow{2}{*}{0.5} & 43 & $93 \quad 36$ & $228 \quad 84$ & $944 \quad 344$ & \\
\hline & $65 \quad 43$ & $152 \quad 92$ & $415 \quad 227$ & 2011951 & \\
\hline & 0.1 & 0.2 & 0.3 & 0.4 & \\
\hline \multicolumn{6}{|c|}{$\alpha=0.001 ; \beta=0.001$} \\
\hline
\end{tabular}

\begin{tabular}{|c|c|c|c|c|c|}
\hline \multicolumn{6}{|c|}{$\alpha=0.01 ; \beta=0.001$} \\
\hline $\mathrm{p}_{p_{0}} p_{1}$ & & 0.2 & 0.3 & 0.4 & 0.5 \\
\hline \multirow{2}{*}{0.1} & & $373 \quad 189$ & 11360 & $56 \quad 31$ & 35 \\
\hline & & $840 \quad 376$ & $219 \quad 114$ & 100 & 56 \\
\hline \multirow{2}{*}{0.2} & $364 \quad 156$ & & $551 \quad 269$ & 152 & 71 \\
\hline & $830 \quad 366$ & & $1314 \quad 560$ & 310 & 132 \\
\hline \multirow{2}{*}{0.3} & $108 \quad 45$ & 544 & & $671 \quad 320$ & 176 \\
\hline & $215 \quad 109$ & $1307 \quad 553$ & & $1634 \quad 681$ & $\begin{array}{ll}364 & 177\end{array}$ \\
\hline \multirow{2}{*}{0.4} & $55 \quad 23$ & $148 \quad 67$ & $667 \quad 306$ & & $\begin{array}{ll}731 & 344\end{array}$ \\
\hline & $97 \quad 54$ & 307 & $1630 \quad 677$ & & $1795 \quad 740$ \\
\hline \multirow{2}{*}{0.5} & 14 & 71 & $174 \quad 80$ & $726 \quad 339$ & \\
\hline & $55 \quad 33$ & 130 & $363 \quad 176$ & $1793 \quad 739$ & \\
\hline & 0.1 & 0.2 & 0.3 & 0.4 & \\
\hline
\end{tabular}




\section{2. Аппроксимация ТОН с использованием аппроксимации}

Большева. В работе Л.Н. Большева [8] была получена формула для аппроксимации биномиального распределения, уточняющая пуассоновскую аппрокимацию при малых значениях $p$ и больших объемах испытаний $n$ :

$$
\mathbf{P}\{T \leqslant m\}= \begin{cases}K(2 y, 2 m+2)+R & \text { при } n \geqslant 2 m+1, \\ 1-K\left(2 y^{\prime}, 2 n-2 m\right)+R & \text { при } n<2 m+1,\end{cases}
$$

где $y=p(2 n-m) /(2-p), y^{\prime}=(m+n+1)(1-p) /(1+p), K(x, \nu)=\mathbf{P}\left\{\chi_{\nu}^{2}>\right.$ $x\}, R=O\left(n^{-2}\right)$ равномерно по всем $0<p<1$, если $m=$ const.

Так как параметры распределения хи-квадрат существенно зависят от $n$, явного выражения квантили, не зависящей от $n$, получить не удается. Но можно вывести серию неравенств, из которых можно получить искомое значение $n$.

Рассмотрим случай $p_{1}+p_{0} \leqslant 1$. В таком случае при достаточно малых значениях $p_{0}$ и $p_{1}$ будет выполняться $n \geqslant 2 C_{n}+1$, и, таким образом, неравенства (1), определяющие критическую константу $C_{n}$ и ТОН $n$ приблизительно будут иметь следующий вид:

$$
\begin{gathered}
1-\mathbf{P}\left\{\frac{2 p_{0}\left(2 n-C_{n}\right)}{2-p_{0}}, 2 C_{n}+2\right\} \leqslant \alpha, \quad 1-\mathbf{P}\left\{\frac{2 p_{0}\left(2 n-C_{n}+1\right)}{2-p_{0}}, 2 C_{n}\right\}>\alpha, \\
\mathbf{P}\left\{\frac{2 p_{1}\left(2 n-C_{n}\right)}{2-p_{1}}, 2 C_{n}+2\right\} \leqslant \beta, \\
\mathbf{P}\left\{\frac{2 p_{1}\left(2 n-2-C_{n-1}\right)}{2-p_{1}}, 2 C_{n-1}+2\right\}>\beta .
\end{gathered}
$$

Простыми преобразованиями получим

$$
\begin{gathered}
n \leqslant \frac{C_{n}}{2}+\frac{2-p_{0}}{4 p_{0}} x\left(\alpha, 2 C_{n}+2\right), \quad n>\frac{C_{n}-1}{2}+\frac{2-p_{0}}{4 p_{0}} x\left(\alpha, 2 C_{n}\right), \\
n \geqslant \frac{C_{n}}{2}+\frac{2-p_{1}}{4 p_{1}} x\left(1-\beta, 2 C_{n}+2\right), \\
n<1+\frac{C_{n-1}}{2}+\frac{2-p_{1}}{4 p_{1}} x\left(1-\beta, 2 C_{n-1}+2\right) .
\end{gathered}
$$

Здесь и далее $x(q, \nu)-q$-квантиль распределения хи-квадрат с $\nu$ степенями свободы и формулы справедливы только при $p_{0}+p_{1} \leqslant 1$. $\mathrm{B}$ противном случае следует $p_{i}$ заменить на $p_{1-i}^{*}=1-p_{i}, i=0,1$, в качестве нулевой гипотезы взять $p_{0}^{*}$, а критическую константу определять каK $n-C_{n}$.

Алгоритм нахождения $n$ и $C_{n}$, удовлетворяющих этим неравенствам, состоит в следующем. Исключив $n$ из первого и третьего неравенств, придем к неравенству

$$
\frac{\left(2-p_{1}\right) p_{0}}{\left(2-p_{0}\right) p_{1}} \leqslant \frac{x\left(\alpha, 2 C_{n}+2\right)}{x\left(1-\beta, 2 C_{n}+2\right)} .
$$


Пусть $c$ - наименьшее $C_{n}$, для которого выполняется это неравенство. Очевидно, что истинное значение $C_{n}$ не меньше $c$. Положим начальное значение $m_{n}$ равным $c$. Из третьего неравенства найдем соответствующее наименьшее $n$, и, используя это значение, из двух первых неравенств определим $m_{n-1}$. Если все неравенства системы выполняются, то полагаем $C_{n}=m_{n}$. В противном случае полагаем $m_{n}=m_{n}+1$ и проводим вычисления $n$ и $m_{n-1}$ заново.

Приведем таблицу значений, полученных данным методом (табл. 2 статьи). Структура таблиц такова: первое значение в каждой ячейке точное значение ТОН нерандомизированного критерия, второе - полученное алгоритмом, описанным в предыдущем абзаце.

Таблица 2. Значения ТОН с использованием аппроксимации Большева

\begin{tabular}{|c|c|c|c|c|c|}
\hline \multicolumn{6}{|c|}{$\alpha=0.05 ; \beta=0.05$} \\
\hline & & 0.2 & 0.3 & 0.4 & 0.5 \\
\hline 0.1 & & $135 \quad 135$ & $\begin{array}{ll}41 & 41 \\
\end{array}$ & $24 \quad 21$ & 13 \\
\hline 0.2 & $202 \quad 200$ & & $204 \quad 208$ & 60 & 28 \\
\hline 0.3 & $\begin{array}{ll}62 & 61\end{array}$ & $295 \quad 304$ & & $248 \quad 259$ & $\begin{array}{ll}67 & 70\end{array}$ \\
\hline 0.4 & $\begin{array}{ll}32 & 31\end{array}$ & $\begin{array}{ll}85 & 86\end{array}$ & $\begin{array}{ll}360 & 378\end{array}$ & & $268 \quad 294$ \\
\hline 0.5 & $19 \quad 19$ & $40 \quad 41$ & $\begin{array}{ll}96 & 101\end{array}$ & $\begin{array}{ll}391 \quad 429\end{array}$ & \\
\hline & 0.1 & 0.2 & 0.3 & 0.4 & \\
\hline
\end{tabular}

\begin{tabular}{|c|c|c|c|c|c|}
\hline \multicolumn{6}{|c|}{$\alpha=0.01 ; \beta=0.05$} \\
\hline$p_{0}$ & & 0.2 & 0.3 & 0.4 & 0.5 \\
\hline 0.1 & & $198 \quad 194$ & $57 \quad 59$ & $30 \quad 30$ & 18 \\
\hline 0.2 & $272 \quad 271$ & & 291297 & $79 \quad 84$ & 37 \\
\hline 0.3 & $83 \quad 82$ & $402 \quad 413$ & & $360 \quad 374$ & $\begin{array}{cc}93 & 101\end{array}$ \\
\hline 0.4 & $\begin{array}{ll}42 \quad 41 \\
\end{array}$ & $111 \quad 114$ & $495 \quad 515$ & & $392 \quad 426$ \\
\hline 0.5 & $25 \quad 25$ & $52 \quad 55$ & 130138 & $535 \quad 587$ & \\
\hline & 0.1 & 0.2 & 0.3 & 0.4 & \\
\hline & & $\alpha=0.01$ & $\beta=0.01$ & & \\
\hline
\end{tabular}

\begin{tabular}{|c|cc|cc|cc|cc|cc|}
\hline \multicolumn{10}{|c|}{$\alpha=0.05 ; \beta=0.05$} \\
\hline$p_{0}$ & $p_{1}$ & & \multicolumn{2}{|c|}{0.005} & \multicolumn{2}{|c|}{0.01} & \multicolumn{2}{|c|}{0.05} & \multicolumn{2}{|c|}{0.1} \\
\hline 0.001 & & & 1829 & 1829 & 628 & 628 & 93 & 94 & 29 & 29 \\
\hline 0.005 & 2910 & 2910 & & & 3137 & 3137 & 124 & 124 & 46 & 46 \\
\hline 0.01 & 1001 & 1001 & 4654 & 4654 & & & 181 & 181 & 61 & 61 \\
\hline 0.05 & 130 & 130 & 198 & 198 & 259 & 259 & & & 298 & 298 \\
\hline 0.1 & 44 & 44 & 64 & 64 & 81 & 81 & 435 & 435 & & \\
\hline$p_{1}$ & 0.001 & 0.005 & 0.01 & 0.05 & & \\
\hline & \multicolumn{10}{|c|}{$\alpha=0.05 ; \beta=0.01$} \\
\hline
\end{tabular}




\begin{tabular}{|c|c|c|c|c|c|}
\hline \multicolumn{6}{|c|}{$\alpha=0.01 ; \beta=0.05$} \\
\hline$p_{p_{0}} p_{1}$ & & 0.005 & 0.01 & 0.05 & 0.1 \\
\hline 0.001 & & $2627 \quad 2627$ & $773 \quad 773$ & $93 \quad 94$ & 46 \\
\hline 0.005 & $3476 \quad 3476$ & & $\begin{array}{ll}4521 & 4521\end{array}$ & $153 \quad 153$ & 61 \\
\hline 0.01 & $1157 \quad 1157$ & $6314 \quad 6314$ & & $234 \quad 234$ & 76 \\
\hline 0.05 & $\begin{array}{ll}130 & 130\end{array}$ & $\begin{array}{ll}229 & 229\end{array}$ & 344 & & $425 \quad 425$ \\
\hline 0.1 & $\begin{array}{ll}64 & 64\end{array}$ & $\begin{array}{ll}81 & 81\end{array}$ & $113 \quad 113$ & $589 \quad 589$ & \\
\hline & 0.001 & 0.005 & 0.01 & 0.05 & \\
\hline \multicolumn{6}{|c|}{$\alpha=0.01 ; \beta=0.01$} \\
\hline
\end{tabular}

Табличные данные свидетельствуют о достаточно высокой точности аппроксимации $n(\alpha, \beta)$, и этот вывод тем более справедлив для малых значений суммы $p_{0}+p_{1}$ тестируемых значений вероятностей.

3. Дефект размера нерандомизированного критерия. Рассмотрим задачу нахождения асимптотики вероятности ошибки первого рода нерандомизированного критерия (размера критерия) при $n \rightarrow \infty$. Пусть $T$ имеет биномиальное распределение с параметрами $(n, p)$ и $F_{n}(x), x \in \mathbf{R},-$ функция распределения нормированной случайной величины $(T-n \mu) / \sigma \sqrt{n}, \mu=p, \sigma^{2}=p(1-p)$. Для аппроксимации $F_{n}$ воспользуемся разложением Эджворта (смысл символа $\approx$ будет объяснен ниже):

$$
\begin{aligned}
F_{n}(x) \approx \widetilde{F}_{n}(x)=\Phi(x)+\varphi(x)( & \frac{\gamma_{1}}{6 \sqrt{n}}\left(x^{2}-1\right)+\frac{\gamma_{2}}{24 n}\left(x^{3}-3 x\right) \\
& \left.+\frac{\gamma_{1}^{2}}{72 n}\left(x^{5}-10 x^{3}+15 x\right)\right),
\end{aligned}
$$

где

$$
\gamma_{1}=\frac{1-2 p}{\sqrt{p(1-p)}}, \quad \gamma_{2}=\frac{1-6 p(1-p)}{p(1-p)}
$$

— коэффициенты асимметрии и эксцесса биномиального $(1, p)$-распределения.

Однако применение разложения Эджворта к решетчатым распределениям обладает рядом существенных особенностей, и это в первую очередь касается точности аппроксимации. Так, в статье Сенатова [9] в теореме 5 и утверждениях на с. 933 и с. 935 устанавливается, что асимптотическая формула

$$
F_{n}(x)=\widetilde{F}_{n}\left(\frac{x-0.5-n \mu}{\sqrt{n} \sigma}\right)+R_{n}, \quad x \in\{0,1, \ldots, n\},
$$

имеет остаточный член $R_{n}$ порядка $O\left(n^{-3 / 2}\right)$ только в точках приведенной решетки $D_{n}=\{0,1, \ldots, n\}$. Вне этой решетки о точности аппроксимации сказать что-либо не представляется возможным. Это является 
ключевым фактом последующего исследования нерандомизированного критерия.

Разложение Корниша-Фишера есть корень уравнения $\widetilde{F}_{n}(x)=1-\alpha$, т.е. разложение Корниша-Фишера имеет отношение только к разложению Эджворта (а не собственно к распределению $F_{n}(x)$ ), и разложение Корниша-Фишера приближает корень этого уравнения с остатком $r_{n}$ порядка $O\left(n^{-3 / 2}\right)$ :

$\widetilde{F}_{n}^{-1}(1-\alpha)=\lambda_{\alpha}+\frac{\gamma_{1}}{6 \sqrt{n}}\left(\lambda_{\alpha}^{2}-1\right)+\frac{\gamma_{2}}{24 n}\left(\lambda_{\alpha}^{3}-3 \lambda_{\alpha}\right)+\frac{\gamma_{1}^{2}}{72 n}\left(\lambda_{\alpha}^{5}-4 \lambda_{\alpha}^{3}+10 \lambda_{\alpha}\right)+r_{n}$,

где $\lambda_{\alpha}=\Phi^{-1}(1-\alpha)$.

Итак, критическая константа $C_{n}(\alpha)$ определяется как наименьшее целое $C$, удовлетворяющее неравенству $\mathbf{P}\{T \geqslant C\} \leqslant \alpha$. Пусть $\widetilde{C}_{n}(\alpha)=$ $\sqrt{n} \sigma \widetilde{F}_{n}^{-1}(1-\alpha)+n \mu$, где $\widetilde{F}_{n}^{-1}(1-\alpha)$ - разложение Корниша-Фишера (9) без остаточного члена. Заметим, что здесь мы опускаем индекс 0 в гипотетическом значении $p_{0}$ параметра $p$, чтобы избежать излишней загруженности в формулах. Положим $\mathbf{C}_{n}(\alpha)=\left\lfloor\widetilde{C}_{n}(\alpha)+0.5\right\rfloor+1$, где $\lfloor x\rfloor-$ целая часть $x$.

Следующая лемма устанавливает асимптотическое разложение для размера критерия $T \geqslant \mathbf{C}_{n}(\alpha)$.

Лемма 1. Для размера критерия $T \geqslant \mathbf{C}_{n}(\alpha)$ справедливо следующее асимптотическое разложение:

$$
\begin{aligned}
& F_{n}\left(\frac{\mathbf{C}_{n}(\alpha)-n \mu}{\sqrt{n} \sigma}\right)=1-\alpha+\varphi\left(\lambda_{\alpha}\right)\left[\frac{1-\left\{\widetilde{C}_{n}(\alpha)+0.5\right\}}{\sqrt{n}}\right. \\
& \left.\quad+\frac{1}{n}\left(\frac{\gamma_{1}^{2}}{72}\left(\lambda_{\alpha}^{5}-4 \lambda_{\alpha}\right)-\frac{\gamma_{1}}{3} \lambda_{\alpha}-\frac{1}{2}\left(\frac{1-\left\{\widetilde{C}_{n}(\alpha)+0.5\right\}}{\sigma}\right)^{2} \lambda_{\alpha}\right)\right] \\
& \quad+O\left(n^{-3 / 2}\right),
\end{aligned}
$$

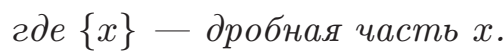

Д о к а з а т е л ь с т в о. В точке $\mathbf{C}_{n}(\alpha)$ значение функции распределения статистики $T$ совпадает со значением функции распределения в точке $\left\lfloor\widetilde{C}_{n}(\alpha)+0.5\right\rfloor+0.5$. Кроме того, согласно вышесказанному, в точке $\left\lfloor\widetilde{C}_{n}(\alpha)+0.5\right\rfloor+0.5$ возможно разложение Эджворта с указанной точностью:

$$
F_{n}\left(\frac{\mathbf{C}_{n}(\alpha)-n \mu}{\sqrt{n} \sigma}\right)=\widetilde{F}_{n}\left(\frac{\left\lfloor\widetilde{C}_{n}(\alpha)+0.5\right\rfloor+0.5-n \mu}{\sqrt{n} \sigma}\right)+O\left(n^{-3 / 2}\right) .
$$

Для того чтобы получить утверждение леммы, достаточно разложить

$$
\widetilde{F}_{n}\left(\frac{\left\lfloor\widetilde{C}_{n}(\alpha)+0.5\right\rfloor+0.5-n \mu}{\sqrt{n} \sigma}\right)=\widetilde{F}_{n}\left(\frac{\widetilde{C}_{n}(\alpha)-n \mu}{\sqrt{n} \sigma}+\frac{1-\left\{\widetilde{C}_{n}(\alpha)+0.5\right\}}{\sqrt{n} \sigma}\right)
$$


в ряд Тейлора в окрестности точки $\left(\widetilde{C}_{n}(\alpha)-n \mu\right) / \sqrt{n} \sigma=F_{n}^{-1}(1-\alpha)$ :

$$
\begin{aligned}
\widetilde{F}_{n}( & \left.\frac{\left\lfloor\widetilde{C}_{n}(\alpha)+0.5\right\rfloor+0.5-n \mu}{\sqrt{n} \sigma}\right) \\
= & \widetilde{F}_{n}\left(\widetilde{F}_{n}^{-1}(1-\alpha)\right)+\widetilde{F}_{n}^{\prime}\left(\widetilde{F}_{n}^{-1}(1-\alpha)\right)\left(\frac{1-\left\{\widetilde{C}_{n}(\alpha)+0.5\right\}}{\sqrt{n} \sigma}\right) \\
& +\frac{1}{2} \widetilde{F}_{n}^{\prime \prime}\left(\widetilde{F}_{n}^{-1}(1-\alpha)\right)\left(\frac{1-\left\{\widetilde{C}_{n}(\alpha)+0.5\right\}}{\sqrt{n} \sigma}\right)^{2} .
\end{aligned}
$$

Остается разложить $\widetilde{F}_{n}$ и ее производные в точке $\widetilde{F}_{n}^{-1}(1-\alpha)$ по степеням $n^{-1 / 2}$. Для этого в $(8)$ положим $x=\widetilde{F}_{n}^{-1}(1-\alpha)$ и разложим каждый член в окрестности точки $\lambda_{\alpha}=\Phi^{-1}(1-\alpha)$. В результате получим

$$
\begin{aligned}
& \widetilde{F}_{n}\left(\widetilde{F}_{n}^{-1}(1-\alpha)\right)=1-\alpha+\varphi\left(\lambda_{\alpha}\right) \frac{\gamma_{1}^{2}}{72 n}\left(\lambda_{\alpha}^{5}-4 \lambda_{\alpha}\right)+O\left(n^{-3 / 2}\right), \\
& \widetilde{F}_{n}^{\prime}\left(\widetilde{F}_{n}^{-1}(1-\alpha)\right)=1-\frac{\gamma_{1}}{3 \sqrt{n}} \lambda_{\alpha}+O\left(\frac{1}{n}\right), \\
& \widetilde{F}_{n}^{\prime \prime}\left(\widetilde{F}_{n}^{-1}(1-\alpha)\right)=\lambda_{\alpha}+O\left(\frac{1}{\sqrt{n}}\right) .
\end{aligned}
$$

После подстановки этих выражений в (11) получаем утверждение леммы.

Таким образом, если $\mathbf{C}_{n}(\alpha)$ совпадает с критической константой критерия $C_{n}(\alpha)$, то (10) представляет собой асимптотическое разложение размера нерандомизированного критерия. Связь между $C_{n}(\alpha)$ и $\mathbf{C}_{n}(\alpha)$ устанавливает следующая теорема.

Теорема 1. Существует такое $N>0$, что постоянная $\mathbf{C}_{n}(\alpha)=$ $\left\lfloor\widetilde{C}_{n}(\alpha)+0.5\right\rfloor+1$ совпадает с критической константой $C_{n}(\alpha)$ рассматриваемого нерандомизированного критерия при всех $n \geqslant N$. Исключение составляет случай

$$
\begin{gathered}
-\frac{\lambda_{\alpha}^{5} \gamma_{1,0}^{2} \sigma_{0}}{72 \sqrt{n}}-\frac{\lambda_{\alpha} \gamma_{1,0}^{2} \sigma_{0} \theta^{2}}{18 \sqrt{n}}+\frac{\lambda_{\alpha}^{6} \gamma_{1,0}^{3} \theta^{2} \sigma_{0}}{216 n}+\frac{\lambda_{\alpha}^{11} \gamma_{1,0}^{4} \theta^{2} \sigma_{0}}{288 n^{3 / 2}} \\
<\left\lfloor\widetilde{C}_{n}(\alpha)\right\rfloor+0.5-\widetilde{C}_{n}(\alpha)<0, \quad \theta \in[0 ; 1],
\end{gathered}
$$

когда $\mathbf{C}_{n}(\alpha)=C_{n}(\alpha)+1$.

Д о к аз ат ель с т в о. Рассмотрим те случаи, когда $\mathbf{C}_{n}(\alpha) \neq$ $C_{n}(\alpha)$. Так как погрешность $R_{n}$ разложения Корниша-Фишера есть величина порядка $O\left(n^{-3 / 2}\right)$, то существует такое $N_{1}$, что $\left|R_{N_{1}}\right|<1$.

$$
\begin{aligned}
\left.1^{0}\right) \quad & F_{n}\left(\frac{\widetilde{C}_{n}(\alpha)-n \mu}{\sqrt{n} \sigma}\right)<1-\alpha, \quad \widetilde{C}_{n}(\alpha)<\left\lfloor\widetilde{C}_{n}(\alpha)\right\rfloor+0.5 \\
& \mathbf{C}_{n}(\alpha)=C_{n}(\alpha)-1 .
\end{aligned}
$$




$$
\begin{array}{ll}
\left.2^{0}\right) \quad & F_{n}\left(\frac{\widetilde{C}_{n}(\alpha)-n \mu}{\sqrt{n} \sigma}\right)>1-\alpha, \quad \widetilde{C}_{n}(\alpha)>\left\lfloor\widetilde{C}_{n}(\alpha)\right\rfloor+0.5 \\
& \mathbf{C}_{n}(\alpha)=C_{n}(\alpha)+1
\end{array}
$$

В случае $\left.1^{0}\right)$, используя равенство (12), получаем

$$
\begin{aligned}
F_{n}\left(\frac{\widetilde{C}_{n}(\alpha)-n \mu}{\sqrt{n} \sigma}\right)= & \widetilde{F}_{n}\left(\frac{\left\lfloor\widetilde{C}_{n}(\alpha)\right\rfloor+0.5-n \mu}{\sqrt{n} \sigma}\right)+O\left(n^{-3 / 2}\right)<1-\alpha \\
= & \widetilde{F}_{n}\left(\frac{\widetilde{C}_{n}(\alpha)-n \mu}{\sqrt{n} \sigma}\right)-\varphi\left(\lambda_{\alpha}\right) \frac{\gamma_{1}^{2}}{72 n}\left(\lambda^{5}-4 \lambda\right) \\
& +O\left(n^{-3 / 2}\right) .
\end{aligned}
$$

Так как $\widetilde{C}_{n}(\alpha)<\left\lfloor\widetilde{C}_{n}(\alpha)\right\rfloor+0.5$, то данное неравенство не может выполняться (за счет слагаемого $\varphi\left(\lambda_{\alpha}\right) \gamma_{1}^{2}\left(\lambda^{5}-4 \lambda\right) /(72 n)$ ) при всех $n$ больше некоторого $N_{2}$. Положим $N=\max \left\{N_{1}, N_{2}\right\}$.

В случае $2^{0}$ ) имеем неравенство, противоположное (13). Для того чтобы выяснить, при каких условиях на значения $\widetilde{C}_{n}(\alpha)$ выполняется это неравенство, разложим

$$
\widetilde{F}_{n}\left(\frac{\left\lfloor\widetilde{C}_{n}(\alpha)\right\rfloor+0.5-n \mu}{\sqrt{n} \sigma}\right)
$$

в ряд Тейлора в точке $\widetilde{F}_{n}^{-1}(1-\alpha)$. В результате получим неравенство

$$
\begin{aligned}
& \frac{1}{\sqrt{n} \sigma}\left(\left\lfloor\widetilde{C}_{n}(\alpha)\right\rfloor+0.5-\widetilde{C}_{n}(\alpha)\right)\left(1-2 \lambda_{\alpha} \frac{\gamma_{1}}{6 \sqrt{n}}\right) \\
& \quad-\frac{1}{2 n \sigma^{2}}\left(\left\lfloor\widetilde{C}_{n}(\alpha)\right\rfloor+0.5-\widetilde{C}_{n}(\alpha)\right)^{2} \lambda_{\alpha}+O\left(n^{-3 / 2}\right) \\
& >-\frac{\gamma_{1}^{2}}{72 n}\left(\lambda_{\alpha}^{5}-4 \lambda_{\alpha}\right)+O\left(n^{-3 / 2}\right) .
\end{aligned}
$$

Найдем те $\left\lfloor\widetilde{C}_{n}(\alpha)\right\rfloor+0.5-\widetilde{C}_{n}(\alpha)$, при которых выполняется это неравенство. Для этого выпишем соответствуюшее квадратное уравнение

$$
\frac{1}{\sqrt{n} \sigma} x\left(1-2 \lambda_{\alpha} \frac{\gamma_{1}}{6 \sqrt{n}}\right)-\frac{\lambda_{\alpha}}{2 n \sigma^{2}} x^{2}=-\frac{\gamma_{1}^{2}}{72 n}\left(\lambda_{\alpha}^{5}-4 \lambda_{\alpha}\right)
$$

относительно переменной $x=\left\lfloor\widetilde{C}_{n}(\alpha)\right\rfloor+0.5-\widetilde{C}_{n}(\alpha)$. Вычисляя дискриминант $\mathscr{D}$ и применяя формулу Тейлора, находим, что

$$
\begin{aligned}
\sqrt{\mathscr{D}} & =\frac{1}{\sqrt{n} \sigma_{0}} \sqrt{1-\frac{4 \lambda_{\alpha} \gamma_{1}}{6 \sqrt{n}}+\frac{\lambda_{\alpha}^{6} \gamma_{1}^{2}}{36 n}} \\
& =\frac{1}{\sqrt{n} \sigma}\left(1-\frac{2 \lambda_{\alpha} \gamma_{1}}{6 \sqrt{n}}+\frac{\lambda_{\alpha}^{6} \gamma_{1}^{2}}{72 n}-\frac{\lambda_{\alpha}^{2} \gamma_{1}^{2} \theta^{2}}{18 n}+\frac{\lambda_{\alpha}^{7} \gamma_{1}^{3} \theta^{2}}{216 n^{3 / 2}}+\frac{\lambda_{\alpha}^{12} \gamma_{1}^{4} \theta^{2}}{288 n^{2}}\right)
\end{aligned}
$$


при некотором $\theta \in[0 ; 1]$. Подставляя это значение в формулу для корней уравнения, получим

$$
\begin{aligned}
\left\lfloor\widetilde{C}_{n}(\alpha)\right\rfloor+0.5-\widetilde{C}_{n}(\alpha)= & -\frac{\lambda_{\alpha}^{5} \gamma_{1}^{2} \sigma}{72 \sqrt{n}}-\frac{\lambda_{\alpha} \gamma_{1}^{2} \theta^{2} \sigma}{18 \sqrt{n}}+\frac{\lambda_{\alpha}^{6} \gamma_{1}^{3} \theta^{2} \sigma}{216 n}+\frac{\lambda_{\alpha}^{11} \gamma_{1}^{4} \theta^{2} \sigma}{288 n^{3 / 2}} \\
\left\lfloor\widetilde{C}_{n}(\alpha)\right\rfloor+0.5-\widetilde{C}_{n}(\alpha)= & 2 \sqrt{n} \sigma+\frac{2 \gamma_{1} \sigma}{3}-\frac{\lambda_{\alpha}^{5} \gamma_{1}^{2} \sigma}{72 \sqrt{n}}-\frac{\lambda_{\alpha} \gamma_{1}^{2} \theta^{2} \sigma}{18 \sqrt{n}} \\
& +\frac{\lambda_{\alpha}^{6} \gamma_{1}^{3} \theta^{2} \sigma}{216 n}+\frac{\lambda_{\alpha}^{11} \gamma_{1}^{4} \theta^{2} \sigma}{288 n^{3 / 2}}
\end{aligned}
$$

Учитывая, что $\left\lfloor\widetilde{C}_{n}(\alpha)\right\rfloor+0.5-\widetilde{C}_{n}(\alpha)<0$, а второй корень уравнения,

$$
-\frac{\lambda_{\alpha}^{5} \gamma_{1}{ }^{2} \sigma}{72 \sqrt{n}}-\frac{\lambda_{\alpha} \gamma_{1}^{2} \sigma \theta^{2}}{18 \sqrt{n}}+\frac{\lambda_{\alpha}^{6} \gamma_{1}^{3} \theta^{2} \sigma}{216 n}+\frac{\lambda_{\alpha}^{11} \gamma_{1}^{4} \theta^{2} \sigma}{288 n^{3 / 2}}<\left\lfloor\widetilde{C}_{n}(\alpha)\right\rfloor+0.5-\widetilde{C}_{n}(\alpha)<0 .
$$

Последние неравенства суть не что иное, как утверждение теоремы. Значение критической константы $C_{n}(\alpha)$ следует из условий $\left.2^{0}\right)$. Кроме того, видно, что случай $\left.2^{0}\right)$ выполняется при близости $\left\{\widetilde{C}_{n}(\alpha)\right\}$ к 0.5 порядка $n^{-1 / 2}$.

Асимптотическое разложение (10) в утверждении леммы 1 можно использовать для построения верхней границы разности между заданным уровнем значимости и размером нерандомизированного критерия при каждом значении $n$.

Следствие 1. Если $n \geqslant N$, то при некотором $R>0$ для дефекта критерия $D(n)=\alpha-\mathbf{P}\left\{T \geqslant C_{n}(\alpha)\right\}$ имеет место неравенство

$$
\begin{aligned}
D(n) \leqslant & \varphi\left(\lambda_{\alpha}\right)\left[\frac{\gamma_{1}^{2}}{72 n}\left(\lambda_{\alpha}^{5}-4 \lambda_{\alpha}\right)+\left(\frac{1}{\sqrt{n} \sigma}\right)\left(1-\frac{\gamma_{1}}{3 \sqrt{n}} \lambda_{\alpha}\right)-\frac{1}{2}\left(\frac{1}{\sqrt{n} \sigma}\right)^{2} \lambda_{\alpha}\right] \\
& +R n^{-3 / 2}
\end{aligned}
$$

Д о к а з а т е л ь с т в о. Утверждение следствия в случае $\mathbf{C}_{n}(\alpha)=$ $C_{n}(\alpha)$ получается применением простейшей оценки $1-\left\{\widetilde{C}_{n}(\alpha)+0.5\right\} \leqslant 1$ к разложению $(10)$. Если $\mathbf{C}_{n}(\alpha)=C_{n}(\alpha)+1$ (случай $\left.2^{0}\right)$ в доказательстве теоремы), то утверждение следствия вытекает из неравенства

$$
\begin{aligned}
1-\alpha & \leqslant F_{n}\left(\frac{C_{n}(\alpha)-n \mu}{\sqrt{n} \sigma}\right)=\widetilde{F}_{n}\left(\frac{\left\lfloor\widetilde{C}_{n}(\alpha)\right\rfloor+0.5-n \mu}{\sqrt{n} \sigma}\right)+O\left(n^{-3 / 2}\right) \\
<F_{n}\left(\frac{\widetilde{C}_{n}(\alpha)-n \mu}{\sqrt{n} \sigma}\right) & =1-\alpha+\varphi\left(\lambda_{\alpha}\right) \frac{\gamma_{1}^{2}}{72 n}\left(\lambda_{\alpha}^{5}-4 \lambda_{\alpha}\right)+O\left(n^{-3 / 2}\right) .
\end{aligned}
$$

Полученный результат проиллюстрирован на рис. 1 для $p_{0}=0.3$ и $\alpha=0.01$. Значение $n$ на оси абсцисс варьируется от 1 до 500, точками отмечены дефекты размера критерия $D(n)$. 


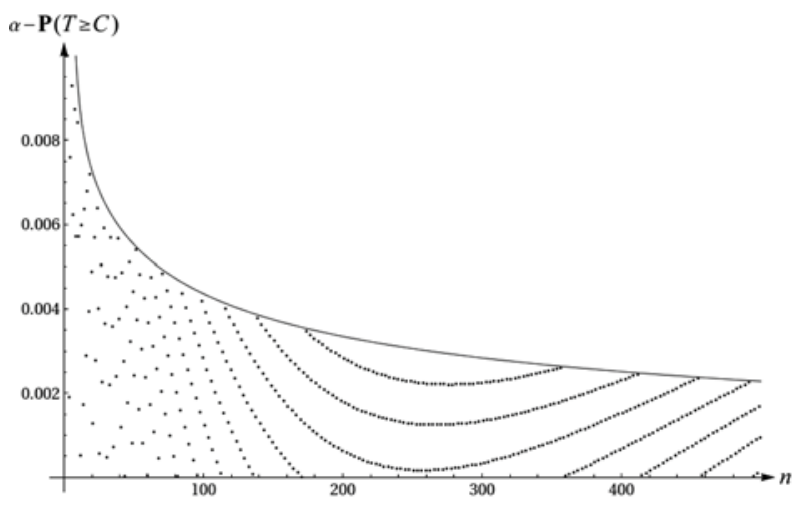

Рис. 1. Дефект размера критерия

В данном примере $N^{*}=1, R<0.25$. Заметим, что случай (13) встречается примерно в $4 \%$ случаев.

4. Асимптотика ТОН с использованием $\mathbf{C}_{n}(\alpha)$. Результаты предыдущего параграфа позволяют построить асимптотику ТОН нерандомизированного критерия, если в качестве критической константы использовать $\mathbf{C}_{n}(\alpha)$, причем $\widetilde{C}_{n}(\alpha)=\sqrt{n} \sigma_{0} \widetilde{F}_{0, n}^{-1}(1-\alpha)+n \mu_{0}$, а $\widetilde{F}_{0, n}^{-1}(1-\alpha)-$ главный член в разложении Корниша-Фишера (9). При этом мы пренебрегаем возможной ошибкой (13) в определении критической константы.

Вероятность ошибки второго рода при критической константе $\mathbf{C}_{n}(\alpha)$ равна

$$
F_{1, n}\left(\frac{\mathbf{C}_{n}(\alpha)-n \mu_{1}}{\sqrt{n} \sigma_{1}}\right)=\widetilde{F}_{1, n}\left(\frac{\left\lfloor\widetilde{C}_{n}(\alpha)+0.5\right\rfloor+0.5-n \mu_{1}}{\sqrt{n} \sigma_{1}}\right)+O\left(n^{-3 / 2}\right) .
$$

Последнее выражение должно удовлетворять условию $F_{1, n}\left(\mathbf{C}_{n}(\alpha)-\right.$ $\left.n \mu_{1} / \sqrt{n} \sigma_{1}\right) \leqslant \beta$. По аналогии с постоянной $\mathbf{C}_{n}(\alpha)$ определим константу $\mathbf{C}_{n}(\beta)=\left\lfloor\widetilde{C}_{n}(\beta)-0.5\right\rfloor+1$, где $\widetilde{C}_{n}(\beta)=\sqrt{n} \sigma_{1} F_{1, n}^{-1}(\beta)+n \mu_{1}$. Объем наблюдений $n(\alpha, \beta)$ определяется как наименьшее $n$, удовлетворяющее неравенству $\mathbf{C}_{n}(\beta) \leqslant \mathbf{C}_{n}(\alpha)$ (см. [5]). Переходя к непрерывной переменной $n$, получаем уравнение для асимптотической оценки ТОН $n(\alpha, \beta)$ :

$$
\widetilde{C}_{n}(\alpha)-\widetilde{C}_{n}(\beta)+1-\left\{\widetilde{C}_{n}(\alpha)+0.5\right\}+\left\{\widetilde{C}_{n}(\beta)-0.5\right\}=0,
$$

где $\{a\}$ означает дробную часть числа $a$.

Положим $\zeta_{n}=1-\left\{\widetilde{C}_{n}(\alpha)+0.5\right\}+\left\{\widetilde{C}_{n}(\beta)-0.5\right\}$. При известном значении $\zeta_{n}$ можно было бы выписать решение (5) из статьи [5], измененное с учетом $\zeta_{n}$ :

$$
\begin{aligned}
\widetilde{n} & =\frac{a^{2}}{\Delta^{2}}+\frac{2 \widetilde{b}}{\Delta}+\frac{2 c a-\widetilde{b}^{2}}{a^{2}}+O(\Delta), \\
a & =\sigma_{0} \lambda_{\alpha}+\sigma_{1} \lambda_{\beta},
\end{aligned}
$$




$$
\begin{aligned}
b= & \frac{1}{6}\left[\left(\lambda_{\alpha}^{2}-1\right) \sigma_{0} \cdot \gamma_{1,0}-\left(\lambda_{\beta}^{2}-1\right) \sigma_{1} \cdot \gamma_{1,1}\right], \\
\widetilde{b}= & b+\zeta_{n} \\
c= & \frac{1}{24}\left[\left(\lambda_{\alpha}^{3}-3 \lambda_{\alpha}\right) \sigma_{0} \cdot \gamma_{2,0}+\left(\lambda_{\beta}^{3}-3 \lambda_{\beta}\right) \sigma_{1} \cdot \gamma_{2,1}\right] \\
& -\frac{1}{72}\left[\left(\lambda_{\alpha}^{5}-4 \lambda_{\alpha}^{3}+10 \lambda_{\alpha}\right) \sigma_{0} \cdot \gamma_{1,0}^{2}+\left(\lambda_{\alpha}^{5}-4 \lambda_{\beta}^{3}+10 \lambda_{\beta}\right) \sigma_{1} \cdot \gamma_{1,1}^{2}\right] .
\end{aligned}
$$

Здесь $a, \widetilde{b}, c$ - коэффициенты в уравнении $(16)$ перед $\sqrt{n}, 1,1 / \sqrt{n}$ соответственно.

Основная теорема статьи [5] утверждает, что для критериев, основанных на статистике, имеющей строго монотонную функцию распределения, можно с помощью формулы (17) аппроксимировать значение ТОН с точностью до единицы. В нашем случае предположение о монотонности функции распределения не выполняется. В то же время это предположение вводилось для определения функции квантили $F_{n}^{-1}(\varepsilon)$. В нашем случае $\mathbf{C}_{n}(\varepsilon)$ однозначно определяет квантиль для любого $0<\varepsilon<1$. Однако это не исчерпывает всех проблем дискретного распределения. Вопервых, функцию $\mathbf{C}_{n}(\varepsilon)$ нельзя считать всюду непрерывной функцией аргумента $n$ (в связи с присутствием $n$ в $\zeta_{n}$ ), что затрудняет использование асимптотических методов обращения уравнения относительно $n$. Во-вторых, уравнение (16) может иметь не одно решение, в том смысле, что для некоторых $n$, больших ТОН, условия (1) могут не выполняться. Однако имеет место следующее утверждение.

Теорема 2. Существует такая положительная константа $\Delta^{*}$, что при всех $\Delta<\Delta^{*}$ значение ТОН $n(\alpha, \beta)$ нерандомизированного критерия не превосходит величинь

$$
\frac{a^{2}}{\Delta^{2}}+\frac{2(b+2)}{\Delta}+\frac{2 c a-(b+2)^{2}}{a^{2}}+1 .
$$

Д о к а з а т е л ь с т в о. Пусть $\zeta_{n}=\zeta$ - фиксированное. Тогда в силу основной теоремы статьи [5] уравнение (16) имеет единственный корень $n^{*}(\zeta)$, полученный с помощью (17) с $\zeta_{n}=\zeta$. При этом $\mid n^{*}(\zeta)-$ $n(\zeta) \mid \leqslant 1$, когда $\Delta<\Delta^{*}(\zeta)$, где $n(\zeta)$ - «истинное» значение ТОН при $\zeta_{n}=\zeta$, а $\Delta^{*}(\zeta)$ - некоторое число, которое, вообще говоря, зависит от $\zeta \in[0 ; 2]$. Однако $[0 ; 2]$ - компакт, и в соответствии $(17) n^{*}(\zeta)$ является непрерывной функцией $\zeta$. Следовательно, можно найти положительную константу $\Delta^{*} \leqslant \Delta^{*}(\zeta)$ при любом $\zeta \in[0 ; 2]$.

Далее, $n^{*}(\zeta)$, будучи многочленом второй степени, достигает своего максимума при

$$
\zeta^{*}=\frac{1}{\Delta a^{2}}-\frac{b}{a^{4}} .
$$

Если учесть, что при уменьшении $\Delta$ переменные $a, b$ ведут себя как 
константы, то $\zeta^{*}>2$ при достаточно малых $\Delta$. Так что заменой $n^{*}(\zeta)$ на меньшее $n^{*}(2)$ получаем утверждение теоремы.

\section{5. Аппроксимация НОВ для рандомизированного критерия} с использованием разложения Корниша-Фишера. Применим метод из статьи И.Н. Володина [5] для нахождения НОВ рандомизированного критерия. Представим функцию распределения $G_{n}$ статистики $\mathfrak{T}=(T+U-n \mu) /(\sqrt{n} \sigma)$ в терминах $F_{n}$ - нормированной функции биномиального распределения:

$$
\begin{aligned}
G_{n}(x) & =\int_{0}^{1} \mathbf{P}\left(\frac{T-n \mu}{\sqrt{n} \sigma}<x-\frac{u}{\sqrt{n} \sigma} \mid U=u\right) d u \\
& =\int_{0}^{1} F_{n}\left(x-\frac{u}{\sqrt{n} \sigma}\right) d u .
\end{aligned}
$$

Заменим под интегралом $F_{n}$ на ее приближение $\widetilde{F}_{n}$ из $(8)$ и воспользуемся разложением Тейлора

$$
\widetilde{F}_{n}\left(x-\frac{u}{\sqrt{n} \sigma}\right) \approx \widetilde{F}_{n}(x)-\frac{u}{\sqrt{n} \sigma} \widetilde{F}_{n}^{\prime}(x)+\frac{1}{2}\left(\frac{u}{\sqrt{n} \sigma}\right)^{2} \widetilde{F}_{n}^{\prime \prime}(x) .
$$

Отсюда после вычисления интеграла получаем, что

$$
G_{n}(x) \approx \widetilde{F}_{n}(x)-\frac{1}{2 \sqrt{n} \sigma} \widetilde{F}_{n}^{\prime}(x)+\frac{1}{6 n \sigma^{2}} \widetilde{F}_{n}^{\prime \prime}(x) .
$$

После вычисления производных получаем, что

$$
\begin{aligned}
G_{n}(x)=\Phi(x)-\varphi(x)( & \frac{1}{\sqrt{n}}\left(\frac{1}{2 \sigma}+\frac{\gamma_{1}}{6}\left(x^{2}-1\right)\right) \\
+\frac{1}{n}( & \frac{x}{6 \sigma^{2}}+\frac{\gamma_{1}}{12 \sigma}\left(x^{3}-3 x\right)+\frac{\gamma_{2}}{24}\left(x^{3}-3 x\right) \\
& \left.\left.+\frac{\gamma_{1}^{2}}{72}\left(x^{5}-10 x^{3}+15 x\right)\right)\right)+O\left(n^{-3 / 2}\right) .
\end{aligned}
$$

Для вычисления обратной функции $G_{n}^{-1}$ теперь уже можно применить разложение Корниша-Фишера. Вывод разложения опирается на разложение Тейлора функции $\Phi(x+(\xi-x))$ относительно точки $x$, где $\xi-$ нормальная $(0,1)$ случайная величина, а $x$ - статистика, относительно которой строится разложение (в нашем случае это $\mathfrak{T})$. Т.е. для справедливости разложения требуется близость распределений $x$ и $\xi$, а используемая в данном случае статистика $\mathfrak{T}$ не является асимптотически нормальной в условиях центральной предельной теоремы. Однако слагаемое $U /(\sqrt{n} \sigma)$ мало при достаточно больших $n$, а значит, ряд Тейлора для $\Phi(x+(\xi-x))$ будет сходиться при достаточно малых $\Delta=p_{1}-p_{0}$. 
Опуская длинные выкладки (более подробно о разложении см., например, [10, п. 6.25]), получим представление

$$
\begin{aligned}
G_{n}^{-1}(\varepsilon)= & \lambda_{\varepsilon}+\frac{1}{\sqrt{n}}\left(\frac{1}{2 \sigma}+\frac{\gamma_{1}}{6}\left(\lambda_{\varepsilon}^{2}-1\right)\right) \\
& +\frac{1}{n}\left(\frac{\lambda_{\varepsilon}}{24 \sigma^{2}}+\frac{\gamma_{2}}{24}\left(\lambda_{\varepsilon}^{3}-3 \lambda_{\varepsilon}\right)+\frac{\gamma_{1}^{2}}{72}\left(\lambda_{\varepsilon}^{5}-4 \lambda_{\varepsilon}^{3}+10 \lambda_{\varepsilon}\right)\right) \\
& +O\left(n^{-3 / 2}\right) .
\end{aligned}
$$

От разложения (9) последнее выражение отличается лишь на величину

$$
\frac{1}{2 \sqrt{n} \sigma}+\frac{\lambda_{\varepsilon}}{24 n \sigma^{2}} .
$$

Выпишем теперь аналог уравнения (16) для рандомизированного критерия. Здесь удобнее будет применить уравнение, использованное в [5] (уравнение немного изменено в целях использования в терминах статистики $\mathfrak{T})$ :

$$
n=\left(\frac{\sigma_{0} G_{0, n}^{-1}(1-\alpha)-\sigma_{1} G_{1, n}^{-1}(\beta)}{\mu_{1}-\mu_{0}}\right)^{2} .
$$

Подставляя полученные разложения (18) для $G_{i, n}^{-1}$ в $(19)$, получаем уравнение для определения асимптотики $\mathrm{HOB}$, аналогичное (4) в [5]. Наконец, используя представление (17), получаем асимптотическое разложение для НОВ:

$$
n^{*}=\frac{a^{2}}{\Delta^{2}}+\frac{2 b}{\Delta}+\frac{2 c^{*} a-b^{2}}{a^{2}}+O(\Delta),
$$

где

$$
c^{*}=c+r, \quad r=\left(\frac{\lambda_{\alpha}}{24 \sigma_{0}}+\frac{\lambda_{\beta}}{24 \sigma_{1}}\right) .
$$

Аналогично работе [8] можно сформулировать следующее утверждение.

Теорема 3. Пусть $n$ - необходимый объем выборки рандомизированного критерия, $n^{*}$ получено из формуль (20). Тогда существует такое число $\Delta^{*}$, что для всех $\Delta=p_{1}-p_{0}<\Delta^{*}$ вьлолняется $\left|n-n^{*}\right| \leqslant 1$.

Судя по численным результатам, границы, полученные здесь, довольно грубо оценивают разницу $n(\alpha, \beta)-n^{*}(\alpha, \beta)$. По всей видимости, $\zeta_{n}$, соответствующее ТОН $n$, убывает вместе с $\Delta$, но медленнее, чем $O(\Delta)$. В численных расчетах для построения адекватной границы $n(\alpha, \beta)-n^{*}(\alpha, \beta)$ было достаточно применить $\zeta \leqslant 0.5$.

Приведем таблицы, демонстрирующие эффект рандомизации в редукции ТОН и точность формулы, полученной в теореме 3 . В левой 
верхней ячейке НОВ (рандомизированный критерий), в правой верхней ячейке ТОН (нерандомизированный критерий), значение внизу получено по формуле (20).

Стоит отметить, что применение разложение Корниша-Фишера остается эффективным даже при различении достаточно разнесенных гипотез (большое значение $\Delta=p_{1}-p_{0}$ ).

Таблица 3. Сравнение рандомизированного и нерандомизированного критериев

\begin{tabular}{|c|c|c|c|c|c|}
\hline \multicolumn{6}{|c|}{$\alpha=0.05 ; \beta=0.05$} \\
\hline$p_{0}$ & & 0.2 & 0.3 & 0.4 & 0.5 \\
\hline 0.1 & & $\begin{array}{ll}134 & 135 \\
134 & \end{array}$ & $\begin{array}{ll}41 & 41 \\
41 & \end{array}$ & $\begin{array}{ll}20 & 24 \\
21 & \end{array}$ & $\begin{array}{ll}13 & 13 \\
13 & \end{array}$ \\
\hline 0.2 & $\begin{array}{ll}200 & 202 \\
199 & \end{array}$ & & $\begin{array}{ll}201 & 204 \\
201 & \end{array}$ & $\begin{array}{ll}56 & 60 \\
55 & \end{array}$ & $\begin{array}{ll}26 & 28 \\
26 & \end{array}$ \\
\hline 0.3 & $\begin{array}{ll}61 & 62 \\
61 & \\
\end{array}$ & $\begin{array}{ll}295 & 295 \\
295 & \\
\end{array}$ & & $\begin{array}{ll}245 & 248 \\
245 & \\
\end{array}$ & $\begin{array}{ll}64 & 67 \\
64 & \\
\end{array}$ \\
\hline 0.4 & $\begin{array}{ll}31 & 32 \\
31 & \end{array}$ & $\begin{array}{ll}82 & 85 \\
81 & \end{array}$ & $\begin{array}{ll}359 & 360 \\
358 & \end{array}$ & & $\begin{array}{ll}267 & 268 \\
267 & \end{array}$ \\
\hline 0.5 & $\begin{array}{ll}19 & 19 \\
19 & \\
\end{array}$ & $\begin{array}{ll}39 & 40 \\
38 & \\
\end{array}$ & $\begin{array}{ll}94 & 96 \\
93 & \\
\end{array}$ & $\begin{array}{ll}389 & 391 \\
389 & \\
\end{array}$ & \\
\hline & 0.1 & 0.2 & 0.3 & 0.4 & \\
\hline
\end{tabular}

\begin{tabular}{|c|c|c|c|c|c|}
\hline \multicolumn{6}{|c|}{$\alpha=0.01 ; \beta=0.05$} \\
\hline$p_{1}$ & & 0.2 & 0.3 & 0.4 & 0.5 \\
\hline 0.1 & & $\begin{array}{ll}192 & 198 \\
195 & \end{array}$ & $\begin{array}{ll}57 & 57 \\
60 & \end{array}$ & $\begin{array}{ll}29 & 30 \\
31 & \end{array}$ & $\begin{array}{ll}18 & 18 \\
20 & \end{array}$ \\
\hline 0.2 & $\begin{array}{ll}269 & 272 \\
271 & \\
\end{array}$ & & $\begin{array}{ll}291 & 291 \\
291 & \end{array}$ & $\begin{array}{ll}79 & 79 \\
80 & \\
\end{array}$ & $\begin{array}{ll}37 & 37 \\
38 & \\
\end{array}$ \\
\hline 0.3 & $\begin{array}{ll}82 & 83 \\
83 & \\
\end{array}$ & $\begin{array}{ll}402 & 402 \\
402 & \end{array}$ & & $\begin{array}{ll}356 & 360 \\
356 & \\
\end{array}$ & $\begin{array}{ll}93 & 93 \\
93 & \\
\end{array}$ \\
\hline 0.4 & $\begin{array}{ll}41 & 42 \\
42 & \\
\end{array}$ & $\begin{array}{ll}111 & 111 \\
111 & \\
\end{array}$ & $\begin{array}{ll}489 & 495 \\
490 & \\
\end{array}$ & & $\begin{array}{ll}389 & 392 \\
388 & \\
\end{array}$ \\
\hline 0.5 & $\begin{array}{ll}25 & 25 \\
26 & \\
\end{array}$ & $\begin{array}{ll}52 & 52 \\
52 & \\
\end{array}$ & $\begin{array}{ll}127 & 130 \\
127 & \\
\end{array}$ & $\begin{array}{ll}534 & 535 \\
533 & \\
\end{array}$ & \\
\hline & 0.1 & 0.2 & 0.3 & 0.4 & \\
\hline
\end{tabular}

Заключение. В статье было рассмотрено три способа аппроксимации НОВ. Общая аналитическая характеризация каждой из формул была дана уже во введении, так что остается подвести суммарный итог численных расчетов. Исследуя данные таблиц, можно прийти к выводу, что наиболее эффективным является применение разложения Корниша-Фишера для квантили статистики рандомизированного критерия. Кроме редукции объема наблюдений эта формула хорошо аппрок- 
симирует само значение НОВ. Кроме того, разложение (18) дает еще и значение критической константы критерия. Аппроксимация НОВ, полученная с использованием теоремы о больших уклонениях, работает неудовлетворительно даже при малых $\alpha$ и $\beta$. Использование аппроксимации хи-квадрат допустимо для $p_{0}<0.1$.

Более существенный результат был получен в отношении размера нерандомизированного критерия. Как оказалось, дефект размера критерия имеет порядок $1 / \sqrt{n}$ (см. следствие 1 ). Кроме того, НОВ нерандомизированного критерия отличается от НОВ рандомизированного критерия не более чем на величину, пропорциональную $1 / \Delta, \Delta=p_{1}-p_{0}$. При этом НОВ рандомизированного критерия имеет главную асимптотику порядка $1 / \Delta^{2}$.

Автор выражает благодарность И.Н. Володину за внимание к работе и полезные обсуждения.

\section{СПИСОК ЛИТЕРАТУРЫ}

1. Novikov An. A., Volodin I. N. Asymptotics of the necessary sample size under small error probabilities. - J. Math. Sci., 1997, v. 84, № 3, p. 1145-1150.

2. Chernov H. Large-sample theory: parametric case. - Ann. Math. Statist., 1956, v. 27 , № 1, p. 1-22.

3. Ширяев А.Н. Вероятность, статистика, случайные процессы, ч. 2. М.: Изд-во Моск. ун-та, 1974, 224 с.

4. Володин И.Н. О числе наблюдений, необходимых для различения двух гипотез о параметре биномиального распределения. - Теория вероятн. и ее примен., 1969, т. 14 , в. 2, с. $327-332$.

5. Володин И. Н. О числе наблюдений, необходимых для различения двух близких гипотез. - Теория вероятн. и ее примен., 1967 , т. 12 , в. 3, с. 575-581.

6. Боровков A. A. Теория вероятности. М.: Наука, 1986, 432 с.

7. Боровков A.A., Могульский A.A. Большие уклонения и проверка статистических гипотез. - Труды Института математики СО РАН, 1993, т. 19, с. 1-222.

8. Большев Л.Н. Асимптотически пирсоновские преобразования. - Теория вероятн. и ее примен., 1963 , т. 8 , в. 2, с. 129-155.

9. Сенатов В.В. О реальной точности аппроксимаций в центральной предельной теореме. - Сиб. матем. журн., 2011, т. 52, № 4, с. 913-935.

10. Кендалл М., Стюарт А. Теория распределений. М.: Наука, 1966, 587 с.

Поступила в редакцию 23.VII. 2012

Исправленный вариант 10.III.2014 